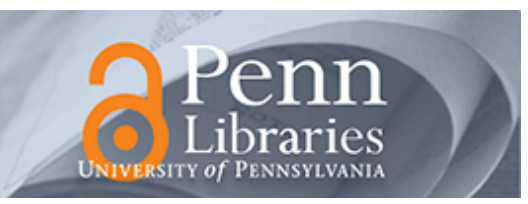

University of Pennsylvania ScholarlyCommons

January 2001

\title{
In-Situ Raman Scattering Studies of Alkali-Doped Single Wall Carbon Nanotubes
}

Agnes Claye

University of Pennsylvania

S. Rahman

University of Pennsylvania

John E. Fischer

University of Pennsylvania, fischer@seas.upenn.edu

A. Sirenko

Pennsylvania State University

G. U. Sumanasekera

Pennsylvania State University

See next page for additional authors

Follow this and additional works at: https://repository.upenn.edu/mse_papers

\section{Recommended Citation}

Claye, A., Rahman, S., Fischer, J. E., Sirenko, A., Sumanasekera, G. U., \& Eklund, P. C. (2001). In-Situ Raman Scattering Studies of Alkali-Doped Single Wall Carbon Nanotubes. Retrieved from

https://repository.upenn.edu/mse_papers/66

Postprint version. Published in Chemical Physics Letters, Volume 333, Issues 1-2, 5 January 2001, pages 16-22.

Publisher URL: http://dx.doi.org/10.1016/S0009-2614(00)01335-X

This paper is posted at ScholarlyCommons. https://repository.upenn.edu/mse_papers/66

For more information, please contact repository@pobox.upenn.edu. 


\title{
In-Situ Raman Scattering Studies of Alkali-Doped Single Wall Carbon Nanotubes
}

\author{
Abstract \\ Electrochemical doping and in-situ Raman scattering were used to study charge transfer in $\mathrm{K}$ - and Li- \\ doped single wall carbon nanotubes (SWNT) as a function of alkali concentration. An $8 \mathrm{~cm}^{-1}$ downshift \\ was observed for the tangential phonon mode of SWNT doped to stoichiometries of $\mathrm{KC}_{24}$ and $\mathrm{Li}_{1} .{ }_{25} \mathrm{C}_{6}$. \\ The shift in both systems is reversible upon de-doping despite an irreversible loss of crystallinity. These \\ results indicate that the tangential mode shifts result from electron transfer from alkali dopants to the \\ SWNT, and that these modes are only weakly affected by long-range order within the ropes.

\section{Comments} \\ Postprint version. Published in Chemical Physics Letters, Volume 333, Issues 1-2, 5 January 2001, pages \\ 16-22. \\ Publisher URL: http://dx.doi.org/10.1016/S0009-2614(00)01335-X \\ Author(s) \\ Agnes Claye, S. Rahman, John E. Fischer, A. Sirenko, G. U. Sumanasekera, and Peter C. Eklund
}




\title{
In-Situ Raman Scattering Studies of Alkali-Doped Single Wall Carbon Nanotubes
}

\author{
A. Claye, S. Rahman, J. E. Fischer \\ Department of Materials Science and Engineering and \\ Laboratory for Research on the Structure of Matter \\ University of Pennsylvania, Philadelphia PA 19104-6272, USA. \\ Sirenko, G. U. Sumanasekera, P. C. Eklund \\ Department of Physics \\ Pennsylvania State University \\ University Park PA 16802-6300, USA.
}

\begin{abstract}
Electrochemical doping and in-situ Raman scattering were used to study charge transfer in K- and Li-doped single wall carbon nanotubes (SWNT) as a function of alkali concentration. An $8 \mathrm{~cm}^{-1}$ downshift was observed for the tangential phonon mode of SWNT doped to stoichiometries of $\mathrm{KC}_{24}$ and $\mathrm{Li}_{1.25} \mathrm{C}_{6}$. The shift in both systems is reversible upon de-doping despite an irreversible loss of crystallinity. These results indicate that the tangential mode shifts result from electron transfer from alkali dopants to the SWNT, and that these modes are only weakly affected by long-range order within the ropes.
\end{abstract}

\section{Introduction}

Single wall carbon nanotubes (SWNT) constitute the newest carbon system in which chemical doping strongly modifies the physical properties [1,2]. The weak Van der Waals bonding between individual nanotubes in a semicrystalline bundle, or "rope", presumably allows for the insertion of dopants in the host lattice, as in graphite intercalation compounds and doped phases of $\mathrm{C}_{60}$. Alkali doping decreases the resistivity of bulk samples by a factor of 30-100 at 300K [1, 3]. A similar result was observed for an individual SWNT rope [4], proving that the enhanced electron transport in bulk samples is an intrinsic property of the ropes. This phenomenon can be explained by valence electron transfer from the alkali atoms into the $\mathrm{C}$ anti-bonding $\pi$ band, which moves the Fermi energy into a region of higher density of states and enhances the conductivity. This mechanism has long been known in graphite intercalation compounds, doped polyacetylene and fullerides. Charge transfer from the alkalis to the nanotubes was proven using Raman scattering, which showed a softening of the tangential vibrational modes for the $\mathrm{C}-\mathrm{C}$ bond upon doping with $\mathrm{K}$ or $\mathrm{Rb}$ [2]. A stiffening of the tangential modes and a decrease in resistivity were observed upon doping with electron acceptors, confirming the amphoteric nature of SWNT $[1,2]$. At this point, however, little is known about the reversibility of these phenomena and their dependence on dopant concentration. One of the limiting factors in this regard is the difficulty of controlling the composition using the vapor phase doping technique. An alternative method is electrochemical doping, which offers precise control of guest stoichiometry and allows for in-situ measurements on the guest-host systems. In-situ Raman scattering and 
electrochemical doping were used to study the insertion mechanism for bisulfate ions in graphite [5,6] and in SWNT [7]. In the present work we used in-situ electrochemical doping and Raman scattering to study the charge transfer in K- and Li-doped SWNT bundles. Raman frequencies were determined vs. dopant concentration, and the reversibility of doping-induced shifts was firmly established.

\section{Experimental}

SWNT were synthesized by pulsed laser vaporization [8], and extensively purified by a series of acid etch and filtration treatments, ending with a vacuum anneal at $1200 \mathrm{C}$ [9].. The samples used in this work consisted of annealed "buckypapers", which are porous, self-standing foils of entangled SWNT ropes. Characterization of the starting material by $\mathrm{x}$-ray diffraction [9] and Raman scattering [9, 10] showed no evidence for nanocrystalline graphite and negligibly small intensity of the D-band at $1350 \mathrm{~cm}^{-1}$ in the undoped materials. We estimate the sample purity to be at least $90 \%$ from a variety of characterization methods, including HRTEM and x-ray diffraction. In-situ Raman scattering and electrochemical doping were performed in a specially designed electrochemical cell made of pyrex tubing with a rectangular cross section attached to a glass-to-metal transition. The cell assembly was performed in an Ar-filled glove box. A buckypaper strip was attached to a stainless steel current collector and used as the working electrode. Alkali metal (K or Li) was smeared on a $\mathrm{Ni}$ mesh and served as the counter and reference electrode. The cell was then filled with electrolyte: $1 \mathrm{M} \mathrm{KCN}$ in triethylborane/tetrahydroduran for $\mathrm{K}$ doping, or $1 \mathrm{M} \mathrm{LiPF}_{6}$ in ethylene carbonate/dimethyl carbonate for Li doping. Electrical contacts were made using $\mathrm{Ni}$ wires that passed through air-tight electrical feedthroughs composed of stainless steel Swagelok fittings with pierced Teflon plugs.

SWNT were doped in the potentiostatic mode (i.e. steps of well-defined potential difference between the sample and the alkali metal counter-electrode) using an EG\&G 273A potentiostat. Raman spectra were measured at the end of each potential step, after waiting for the current to fall below $1 \mu \mathrm{A} / \mathrm{mg}$ to ensure quasi-equilibrium conditions. Each cell was cycled for at least one full cycle in order to assess the reversibility of the charge transfer.

Raman scattering was performed at room temperature using a SPEX Triplemate 1877 spectrometer (resolution $\sim 0.7 \mathrm{~cm}^{-1}$ ) equipped with a liquid nitrogen cooled chargecoupled-device detector. The $514.5 \mathrm{~nm}(2.4 \mathrm{eV})$ line of an $\mathrm{Ar}^{+}$ion laser was used for excitation. To avoid heating of the sample, the pumping power density was limited to 10 $\mathrm{mW} / \mathrm{mm}^{2}$. Experiments were performed using a near Brewster-angle backscattering geometry. The polarization of the incident and scattered light was perpendicular to the plane of incidence.

\section{Results and discussion}

Figure 1(a) presents the cell voltage vs. electrochemical charge $Q$ for a galvanostatic cycle on a K/SWNT cell. Note that the doping is fully reversible, albeit hysteretic. Assuming that one electron flowing in the electrochemical circuit corresponds to the insertion of one alkali ion into a SWNT rope (a safe assumption in the case of potassium but not lithium; see below), then $Q$ is identical to the $\mathrm{K} / \mathrm{C}$ ratio. The maximum $\mathrm{K}$ 
uptake is $\mathrm{K} / \mathrm{C}=0.04$, corresponding to about $\mathrm{KC}_{24}$. This is less than that obtained with vapor doping which yields about $\mathrm{KC}_{8}$ or $\mathrm{K} / \mathrm{C} \sim 0.12$ at saturation [1]. The lower $\mathrm{K}$ uptake using electrochemical doping is most likely due to co-insertion of THF which partially solvates the $\mathrm{K}$ ions [11]. This is known to occur in graphite using the same electrolyte, for which the K-saturated phase is the ternary compound $\mathrm{K}(\mathrm{THF})_{2} \mathrm{C}_{24}$ rather than the $\mathrm{KC}_{8}$ saturated binary obtained by vapor doping [12]. Potassium insertion in SWNT is entirely reversible, since the same charge is obtained upon reduction and oxidation between the same voltage limits in Figure 1(a). No voltage plateau is observed in the charge/discharge curve, indicating that there is no well-defined potential for $\mathrm{K}$ insertion or removal in SWNT. We previously attributed these steep voltage profiles to the absence of well-defined intercalation sites in SWNT, in contrast with graphite, polyacetylene and $\mathrm{C}_{60}$ [13]. We believe that the large dispersity in SWNT chirality, diameter and length in real SWNT samples prevents the formation of ordered doped phases, yielding a distribution of potentials for alkali insertion in the SWNT host rather than the well-defined redox potentials characteristic of truly crystalline host materials [11].

Figure 2 presents a series of Raman spectra for K-doped SWNT at various K/C ratios from 0 (curve a) to 0.040 (curve f), obtained by cross-referencing the constant potentials during in situ doping with the potentials obtained from separate galvanostatic (chargedischarge) measurements. The currents were $5-20 \mu \mathrm{A}$ for $\sim 1 \mathrm{mg}$ working electrodes to ensure quasi-equilibrium conditions. The spectrum for the pristine sample in Figure 2 is dominated by two first-order bands at $1593.5 \mathrm{~cm}^{-1}$ and $1567 \mathrm{~cm}^{-1}$, which have been identified respectively as the $\mathrm{E}_{1 \mathrm{~g}}$ (or $\mathrm{A}_{1 \mathrm{~g}}$ ) and the $\mathrm{E}_{2 \mathrm{~g}}$ modes for isolated tubes [14]. The broad features in the frequency range $1300-1530 \mathrm{~cm}^{-1}$ are due to Raman scattering from the electrolyte. These features obscure the possible observation of a disorder-induced Dband centered at $1350 \mathrm{~cm}^{-1}$. A large luminescent background, which we ascribe to the electrolyte, was also observed. Upon electrochemical doping, we observed a shift and a continuous broadening of the two tangential modes. This shift is fully reversible upon de-doping. There are also some changes in the $1350-1400 \mathrm{~cm}^{-1}$ region. These are difficult to interpret due to the obscuring effect of the electrolyte signal.

In order to quantify the doping-induced shifts, we modeled the tangential modes at each $\mathrm{K} / \mathrm{C}$ value using two Lorentzian lines. While five lines have been assigned to these modes in pristine SWNT [14], two were sufficient to fit the data in Figure 2. Three constraints were applied to the fits. First, we maintained the pristine $26.5 \mathrm{~cm}^{-1}$ separation between the two lines at all compositions, assuming that all modes are equally affected by charge transfer. Second, the two lines were constrained to broaden equally. Finally, the intensity ratio of the two bands was forced to be constant, assuming that doping changes the resonance conditions equally for different modes. The fitting results are presented in Figure 1(b), in which the Raman shift for the highest frequency band is plotted as a function of $\mathrm{K} / \mathrm{C}$. The shift was found to be non-monotonic with charge. At very low doping levels $(\mathrm{K} / \mathrm{C}<0.005)$, a downshift $\left(\sim 1.5 \mathrm{~cm}^{-1}\right)$ was observed. Then for $\mathrm{K} / \mathrm{C}$ ratios between 0.005 and 0.01 , an upshift was seen, which saturated at $\sim 4 \mathrm{~cm}^{-1}$. Finally, between 0.015 and 0.04 , a continuous $\sim 9 \mathrm{~cm}^{-1}$ downshift took place. The nonmonotonic behavior at very low doping levels is not well understood. In-situ dc 4-probe 
and in-situ microwave conductivity of $\mathrm{KC}_{\mathrm{x}}$ in an electrochemical cell exhibited a continuous decrease in resistance over the entire range of compositions between pristine SWNT and $\mathrm{KC}_{24}$ [11], suggesting a continuous charge transfer between $\mathrm{K}$ and $\mathrm{C}$. While the $4 \mathrm{~cm}^{-1}$ value for the upshift might be influenced by the constraints of our fits, Figure 2 shows at least qualitatively that this upshift is real. The same effect was observed at low doping levels by Iwasa et al. for vapor-doped K-SWNT [15]. We interpret the continuous and linear downshift $\Delta \omega$ for $\mathrm{K} / \mathrm{C}>0.015$ as evidence for valence electron transfer from $\mathrm{K}$ atoms to the $\mathrm{C} \pi$ anti-bonding band, which lengthens the $\mathrm{C}-\mathrm{C}$ bond and softens the tangential vibrational modes, similar to the behavior observed in graphite intercalation compounds [16,17]. A straight line can be fit to the data for $0.015<\mathrm{K} / \mathrm{C}$ $<0.04$ from which we obtain $\Delta \omega / \Delta \mathrm{Q} \sim 370 \mathrm{~cm}^{-1} / \mathrm{electron}$ per $\mathrm{C}$, close to the value $\sim 340 \mathrm{~cm}^{-1} /$ electron per $\mathrm{C}$ observed in alkali doped $\mathrm{C}_{60}$ [18] but larger than the value $\sim 280 \mathrm{~cm}^{-1}$ /electron per $\mathrm{C}$ observed in $\mathrm{KC}_{8}$ graphite [16,17]. Applying our value to the shift measured on $\mathrm{K}$ vapor-doped SWNT [2] implies a composition of $\mathrm{KC}_{8.3}$ for that sample, consistent with the saturation weight uptake using the same synthesis conditions [1].

The SWNT bands in Figure 2 broaden continuously with $\mathrm{K}$ doping. This phenomenon has also been observed in other donor and acceptor compounds of SWNT [2]. The line broadening could either be due to a disordered distribution of dopants, as would be implied by inhomogeneous doping [11], or to an increase in the electron-phonon interaction, the latter being a homogeneous line-broadening mechanism. The shift and broadening are entirely reversible upon electrochemical de-doping, as seen in Figures 2 and 1(b). The de-doped and pristine spectra are very similar, also indicating that the charge transfer mechanism is fully reversible. This result is consistent with in-situ 4-probe resistance, which showed that the 15-fold drop in $\mathrm{R}$ upon doping is fully recovered upon de-doping [11]. In-situ X-ray diffraction during electrochemical doping/de-doping also showed that $\mathrm{K}$ insertion is accompanied by an irreversible crystallinity loss in the rope lattice [11]. The full recovery of the Raman shift, despite the doping-induced structural disorder in the bundles, suggests that inter-tube long-range order has little or no effect on the tangential modes. The fact that the linewidths decrease almost to the pristine values upon de-doping confirms that the broadening mechanism is not due to long-range structural disorder, but rather to local disorder in the dopant distribution and/or electron-phonon coupling.

The solid state electrochemistry of the SWNT/Li system is more complicated, as reported earlier in detail [13,19]. Figure 3(a) presents voltage profiles for a galvanostatic cycle of a Li/SWNT cell. The first half-cycle exhibits a large irreversible plateau at $0.9 \mathrm{~V}$ due to the reduction of the ethylene carbonate/dimethyl carbonate electrolyte which forma a surface passivation layer on the carbon electrode. One component of this amorphous, polymeric film is $\mathrm{LiCO}_{3}$, so its formation represents a parasitic and irreversible consumption of Li. This phenomenon has been extensively studied and takes place for all carbons reduced in this specific electrolyte [20]. Since it is difficult to distinguish the charge consumed in this irreversible process from that representing actual SWNT doping, the Li/C ratios in the SWNT upon first doping cannot be precisely determined and in general $\mathrm{Q}$ is no longer equal to the $\mathrm{M} / \mathrm{C}$ ratio. A lower bound on $\mathrm{Li} / \mathrm{C}$ 
for the saturated phase can be estimated from the first de-doping half-cycle since the parasitic Li uptake is irreversible. This gives $450 \mathrm{mAh} / \mathrm{g}$, or $\mathrm{Li} / \mathrm{C}=0.21$, slightly greater than the 0.17 corresponding to the saturated phase $\mathrm{LiC}_{6}$ in graphite [21]. Using electrochemical doping, the maximum reversible Li density in SWNT is therefore at least five times greater than for K since there is no co-intercalated solvent. Similar to $\mathrm{K} / \mathrm{SWNT}$, there is no well-defined potential for $\mathrm{Li}$ insertion and removal, ruling out the possibility of staging via well-defined interstitial sites.

Figure 4 presents a series of Raman spectra for Li-doped SWNT after doping at various constant potentials. The pristine spectrum is dominated by the same two first-order maxima described above. The feature at $1485 \mathrm{~cm}^{-1}$ is Raman scattering from the electrolyte. A strong luminescent background was also observed, which we ascribe to the electrolyte. On the other hand, and in contrast to the KCN/THF electrolyte, the D-band region centered at $1350 \mathrm{~cm}^{-1}$ is not obscured prior to doping. Upon doping between $3 \mathrm{~V}$ and $0 \mathrm{~V}$, we again observe a continuous shift and broadening of the two SWNT tangential modes. Between $3 \mathrm{~V}$ and $1.2 \mathrm{~V}$, an additional line at $1350 \mathrm{~cm}^{-1}$ progressively emerges. This line, or D-band, is generally characteristic of disorder-induced scattering by phonons at the zone edge, and is dominant in samples of nanocrystalline carbons.

In the present case we can safely assume that it is not related to the passivation layer since the latter starts forming at about $0.9 \mathrm{~V}$ while the D- band emerges between 3 and $1.2 \mathrm{~V}$. Furthermore, it is not removed by dedoping and washing in water (spectrum $\mathrm{g}$ in Figure 4).

In order to quantify the doping-induced changes in the tangential modes, we modeled the Raman spectra at all doping levels using the procedure described above, i.e., two Lorentzian lines shifting and broadening equally with doping. The fitting results are presented in Figure 3(b) as a function of charge for one full doping/dedoping cycle between $3 \mathrm{~V}$ and $0 \mathrm{~V}$. Upon doping, we observed a monotonic but nonlinear downshift of both modes, by $8 \mathrm{~cm}^{-1}$ at saturation. Note in particular that the doping curve in

Figure 3(b) is qualitatively similar to the voltage profile in Figure 3(a), namely there is no Raman shift through the charge interval corresponding to the formation of the passivation layer (the long plateau between 100 and $700 \mathrm{~mA} \cdot \mathrm{h} / \mathrm{g}$ ). This suggests that the passivation layer has little influence on the properties of individual nanotubes. The slope of the Raman shift vs. charge in Figure 3(b) decreases with charge. At low doping levels, we find $\Delta \omega / \Delta \mathrm{Q} \sim 100 \mathrm{~cm}^{-1} /$ electron per $\mathrm{C}$ atom, while at higher doping levels we find $\Delta \omega / \Delta \mathrm{Q} \sim 5-10 \mathrm{~cm}^{-1} /$ electron per $\mathrm{C}$ atom. We previously showed that most of the resistance drop in bulk SWNT upon Li doping occurs at very low doping levels [11], consistent with the Raman results. This could be explained by a concentration-dependent specific charge transfer. For example, it is possible that at low doping levels the charge transfer is complete (i.e. formally $\mathrm{Li}^{+}$in the SWNT host), becoming only partial (mixed ionic-covalent Li-C bonding) as the Li density in the SWNT host increases. This would also explain why the Raman shift in saturated Li/SWNT is equal to that in saturated K/SWNT, despite the much larger alkali concentration in the former compound.

The charge transfer in Li-SWNT was found to be only partly reversible electrochemically, in contrast to K/SWNT. Upon de-doping, we observed only a $3 \mathrm{~cm}^{-1}$ upshift over a $450 \mathrm{~mA} . \mathrm{h} / \mathrm{g}$ charge interval (x's in Figure 3b), and a very slight narrowing 
of the two modes. We subsequently took the sample out of the electrochemical cell and washed it in de-ionized water and ethanol in order to remove the remaining Li (filled square in Figure 3b). The Raman spectrum for the washed sample is presented at the top of Figure 4. It is very similar to that of the pristine material, although the bands are slightly broader. This suggests that Li cannot be entirely removed by electrochemically oxidizing the sample up to $3 \mathrm{~V}$, but it can be removed with a more aggressive oxidizing treatment. This result is consistent with the reported decreasing reversible Li capacity as a function of cycling, which suggests that some Li remains trapped in the $\mathrm{C}$ host on each cycle [13]. It is also consistent with the in-situ resistance results, which show that the resistance for the sample at $3 \mathrm{~V}$ decreases with the number of cycles [13]. All these findings suggest that the electrochemical reaction is not fully reversible between $3 \mathrm{~V}$ and $0 \mathrm{~V}$. A large hysteresis is observed in the voltage profiles between charge and discharge, so we expect to improve the reversibility by using a larger potential limit upon oxidation. The $3 \mathrm{~V}$ limit was chosen because of electrolyte breakdown at higher potentials, thus a different choice of electrolyte would be needed to achieve higher voltages.

In-situ X-ray diffraction and electrochemical doping showed that electrochemical Li insertion yields an irreversible crystallinity loss in the rope lattice [13]. The recovery of the Raman shift upon washing, despite the induced structural disorder, again suggests that long-range order within SWNT ropes has a small effect on the tangential phonon modes. The fact that the lines sharpen, almost to the pristine values, upon de-doping confirms that the line broadening mechanism is not due to defects on individual nanotubes or in the rope lattice, but either to the random location of the dopants or to enhanced electronphonon interactions as the sample becomes more metallic.

The growth of the D-band upon doping was found to be irreversible upon electrochemical de-doping and washing. This further rules out the passivation layer as the origin of the doping-induced D-band since the carbonate is water soluble. The persistence of the Dband after dedoping and washing is therefore probably associated with some form of doping-induced disorder or formation of stable defects. Similar conclusions cannot be drawn from the potassium data due to the obscuring effect of the electrolyte, thus there is no evidence that $\mathrm{K}$ and $\mathrm{Li}$ behave differently as regards doping-induced defects or disorder. In both cases the crystallinity of the rope lattice is completely suppressed by doping to saturation.

Interpreting the doping-induced Raman shifts is complicated by the fact that our pristine samples consist of an unknown mixture of metallic and semiconducting tubes. One is tempted to assume that semiconducting tubes dominate the initial doping response since the Fermi energy shift per added carrier should be larger than for the metallic ones [22]. This needs to be verified, for example by excluding the presence of internal electrostatic barriers between tubes with different electronic properties in the pristine state.

\section{Conclusion}

Electrochemical doping allowed us to study the Raman scattering in alkali-doped SWNT as a function of composition, to the best of our knowledge for the first time. We demonstrated the reversible charge transfer between the dopants and the host, which yields a softening of the tangential vibrational phonon modes, similar to observations in 
graphite intercalation compounds and fullerides. For potassium, the shift per charge per $\mathrm{C}$ atom is much closer to the corresponding fulleride than to the GIC. The shift is fully reversible in $\mathrm{K}$ and Li-doped SWNT, although electrochemical oxidation is not sufficient to remove all the Li in SWNT. The Raman frequency of the tangential phonon modes was found to be insensitive to the degree of long-range order in the rope lattice.

\section{Acknowledgments}

Purified SWNT were provided by A. G. Rinzler and R. E. Smalley. Work at the University of Pennsylvania was funded by the National Science Foundation MRSEC Program, grant No. DMR96-32598 and by New Energy Development Organization. Work at the University of Kentucky and the Pennsylvania State University was funded by the National Science Foundation MRSEC Program, grant No. DMR98-09686 and by the Department Of Defense DEPSOR Program, grant No. DAAB07-97-CJ036.

\section{References}

[1] R. S. Lee, H. J. Kim, J. E. Fischer, A. Thess, R. E. Smalley, Nature 388 (1997) 255.

[2] A. M. Rao, P. C. Eklund, S. Bandow, A. Thess, R. E. Smalley, Nature 388 (1997) 257.

[3] L. Grigorian, G. U. Sumanasekera, A. L. Loper, S. Fang, J. L. Allen, P. C. Eklund, Phys. Rev. B 58 (1998) R4195.

[4] R. S. Lee, H. J. Kim, J. E. Fischer, J. Lefebvre, M. Radosavljevic, J. Hone, A. T. Johnson, Phys. Rev. B 61 (2000) 4526.

[5] A. Metrot, J. E. Fischer, Synthetic Metals 3 (1981) 201.

[6] P. C. Eklund, in Intercalation in Layered Solids, ed. By M. S. Dresselhaus, Plenum Press, NY (1986), p.313.

[7] G. U. Sumanasekera, J. L. Allen, S. L. Fang, A. L. Loper, A. M. Rao, P. C. Eklund, J. Phys. Chem 103 (1999) 4292.

[8] A. Thess, R. Lee, P. Nikolaev, H. Dai, P. Petit, J. Robert, C. Xu, Y. H. Lee, S. G. Kim, A. G. Rinzler, D. T. Colbert, G. E. Scuseria, D. Tomanek, J. E. Fischer, R. E. Smalley, Science 273 (1996) 483.

[9] A. G. Rinzler, J. Liu, H. Dai, P. Nikolaev, C. B. Huffman, F. J. Rodriguez-Macias, P. J. Boul, A. H. Lu, D. Heymann, D. T. Colbert, R. S. Lee, J. E. Fischer, A. M. Rao, P. C. Eklund, R. E. Smalley, Appl. Phys. A-Mater. 67 (1998) 29.

[10] A. M. Rao, private communication.

[11] A. Claye, N. Nemes, A. Janossy, J. E. Fischer, Phys. Rev. B (2000).

[12] M. F. Quinton, A. P. Legrand, F. Beguin, Synthetic Metals 13 (1986) 179.

[13] A. Claye, J. E. Fischer, C. B. Huffman, A. G. Rinzler, R. E. Smalley, J.

Electrochem. Soc. (August 2000).

[14] A. M. Rao, E. Richter, S. Bandow, B. Chase, P. C Eklund, K. A Williams, S. Fang, K. R. Subbaswamy, M. Menon, A. Thess, R. E. Smalley, G. Dresselhaus, M. S. Dresselhaus, Science 275 (1997) 187.

[15] Y. Iwasa, H. Fudou, T. Yatsu, T. Mitani, Y. Achiba, H. Kataura, APS meeting abstract R 10.002, March 2000.

[16] P. C. Eklund, G. Dresselhaus, M. S. Dresselhaus, J. E. Fischer, Phys. Rev. B 16 (1977) 3330.

[17] M. S. Dresselhaus. G. Dresselhaus, Adv. Phys. 30 (1981) 139.

[18] H. Kuzmany, M. Matus, B. Burger, J. Winter, Adv. Mater. 6 (1994) 731. 
[19] B. Gao, A. Kleinhammes, X. P. Tang, C. Bower, L. Fleming, Y. Wu, O. Zhou, Chem. Phys. Lett. 307 (1999) 153.

[20] D. Aurbach, Y. Ein-Eli, B. Markovsky, A. Zaban, S. Luski, Y. Carmeli, H. Yamin, J. Electrochem. Soc. 142 (1995) 2882.

[21]. D. Guerard, A. Herold, Carbon 13 (1975) 337.

[22]. S. Kazaoui, N. Minami, R. Jacquemin, H. Kataura and Y. Achiba, Phys. Rev. B 60, 13339 (1999).

\section{Figure captions}

Figure 1 (a) Galvanostatic charge/discharge for a SWNT/K cell in 1M KCN triethylborane/tetrahydrofuran. Fully reversible behavior is observed, with a small hysteresis. (b) Fitting results for the in situ Raman spectra of K-doped SWNT. The Raman shift for the high frequency tangential mode is represented as a function of stoichiometry upon doping (open circles) and complete de-doping (one filled diamond).

Figure 2: In-situ Raman spectra for K-doped SWNT at various K/C ratios. Starting from the bottom: (a) pristine sample, $\mathrm{K} / \mathrm{C}=0$; b) 0.002 ; c) 0.004 ; d) 0.020 ; e) 0.034 ; f) 0.040; g) (top) electrochemically de-doped, $\mathrm{K} / \mathrm{C}=0$.

Figure 3 (a) Galvanostatic charge/discharge for a SWNT/Li cell in $1 \mathrm{M} \mathrm{LiPF}_{6}$ ethylene carbonate/dimethyl carbonate. The long plateau between 100 and $700 \mathrm{mV}$ corresponds to the parasitic Li uptake associated with electrolyte decomposition (see text). (b) Fitting results for the Raman spectra of Li-doped SWNT. The Raman shift for the high frequency mode is represented as a function of charge upon doping (open circles), partial de-doping ( x x x ) and washing in water and ethanol (single filled square).

Figure 4: In-situ Raman scattering spectra for Li-doped SWNT at various potentials: (a) pristine sample; (b) $2 \mathrm{~V}$; (c) $1.5 \mathrm{~V}$; (d) $0.8 \mathrm{~V}$; (e) $0.02 \mathrm{~V}$; (f) electrochemically de-doped to $3 \mathrm{~V} ;(\mathrm{g})$ electrochemically de-doped and washed in water and ethanol. 
Figure 1

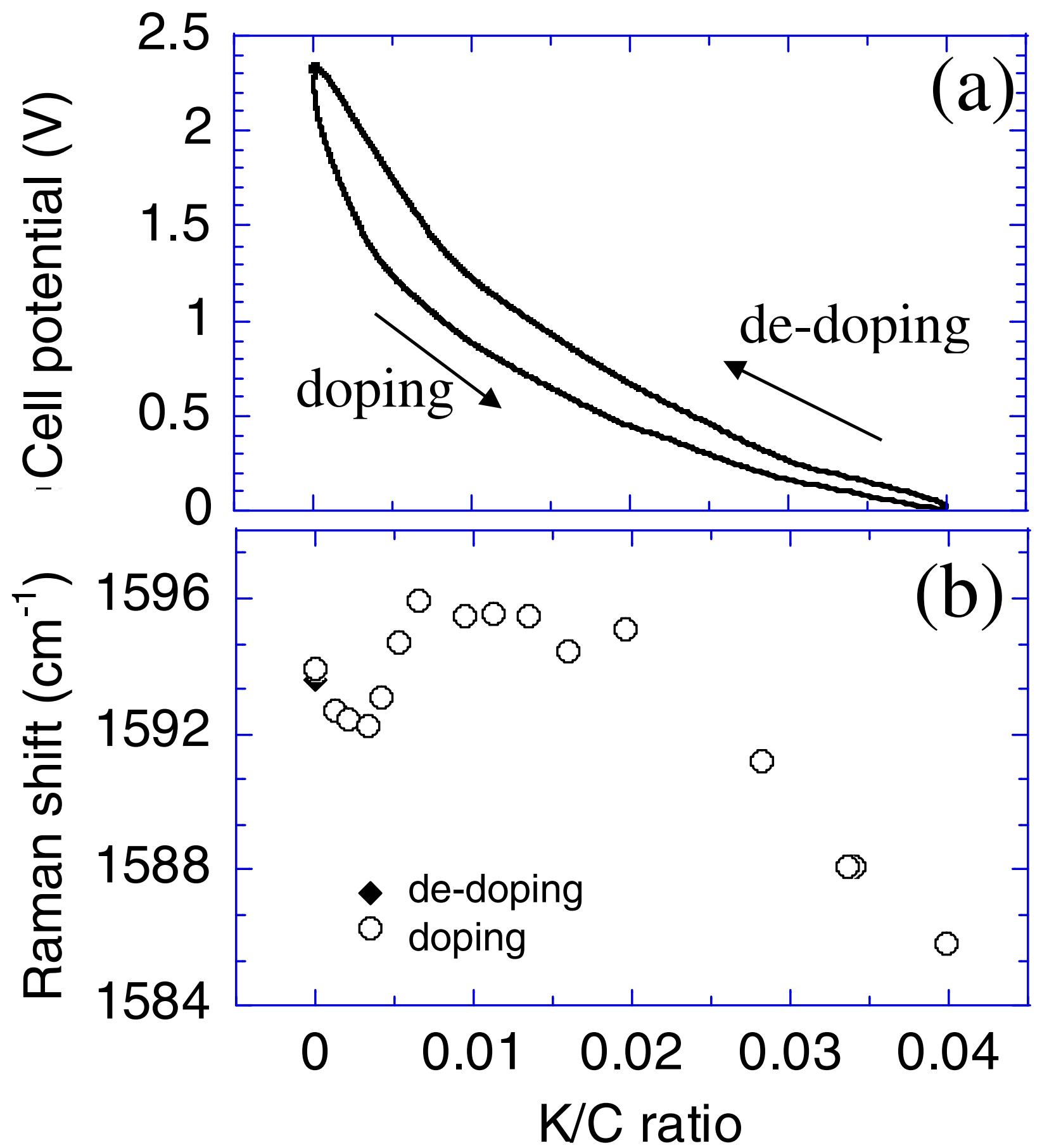


Figure 2

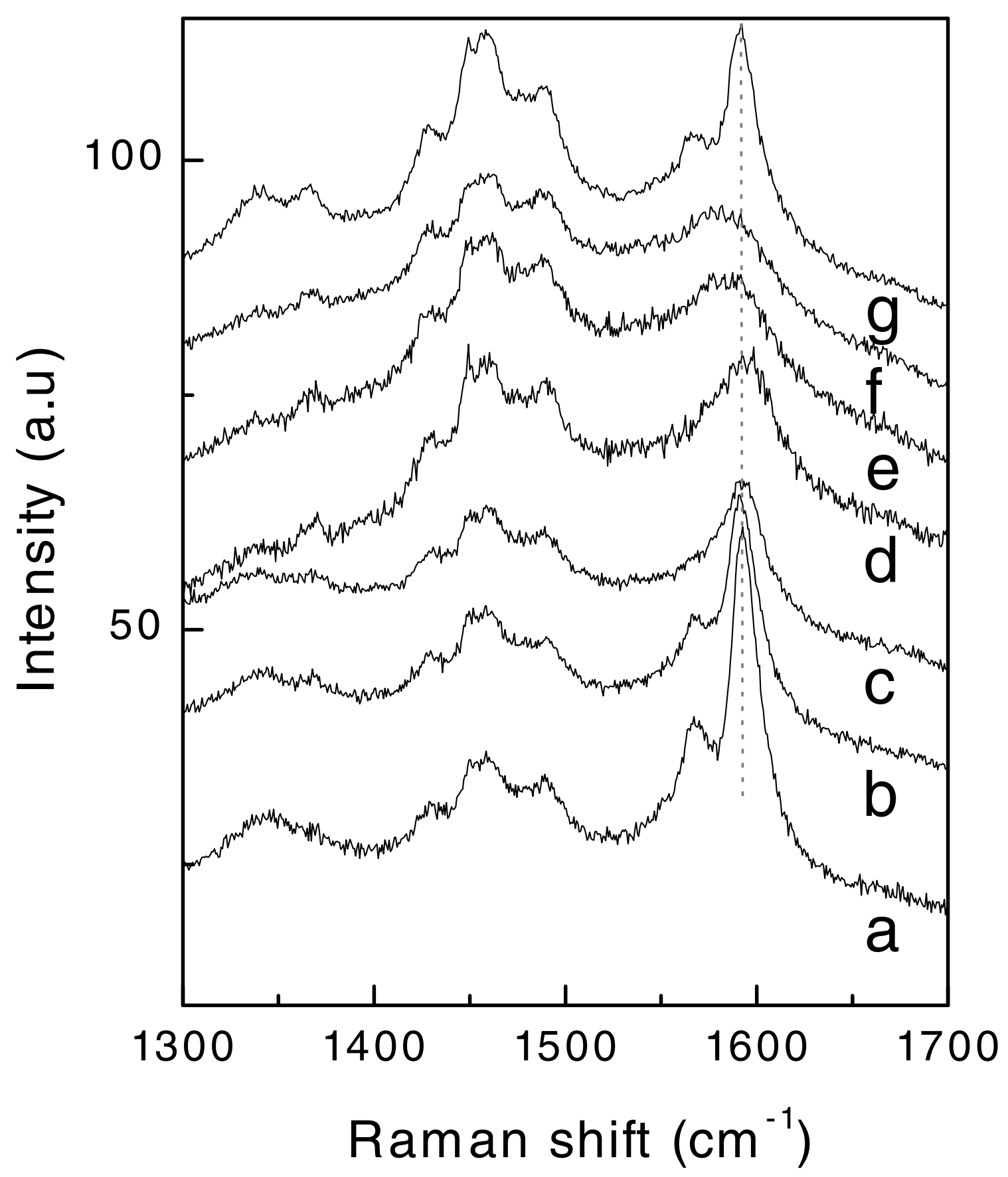


Figure 3

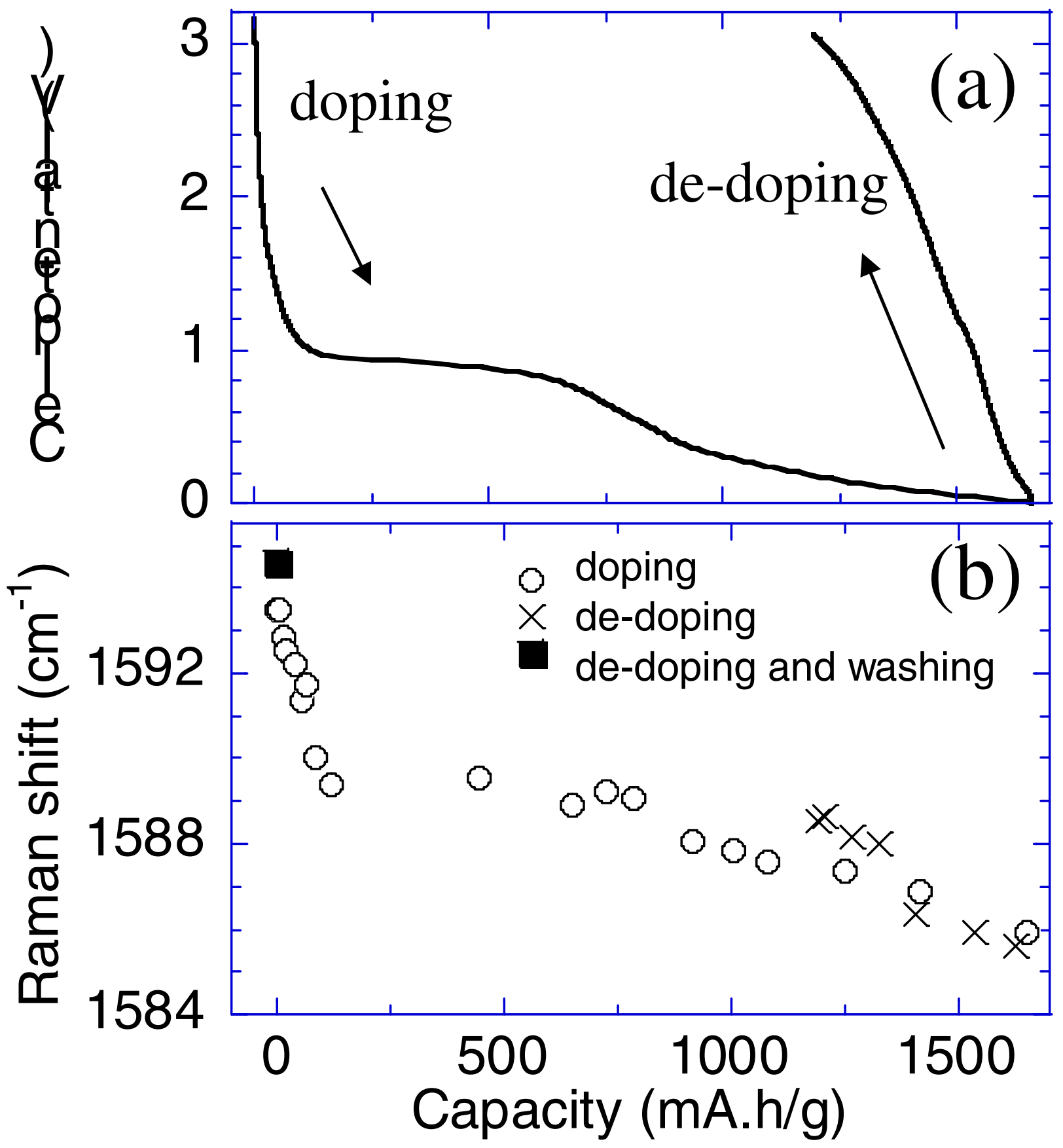


Figure 4

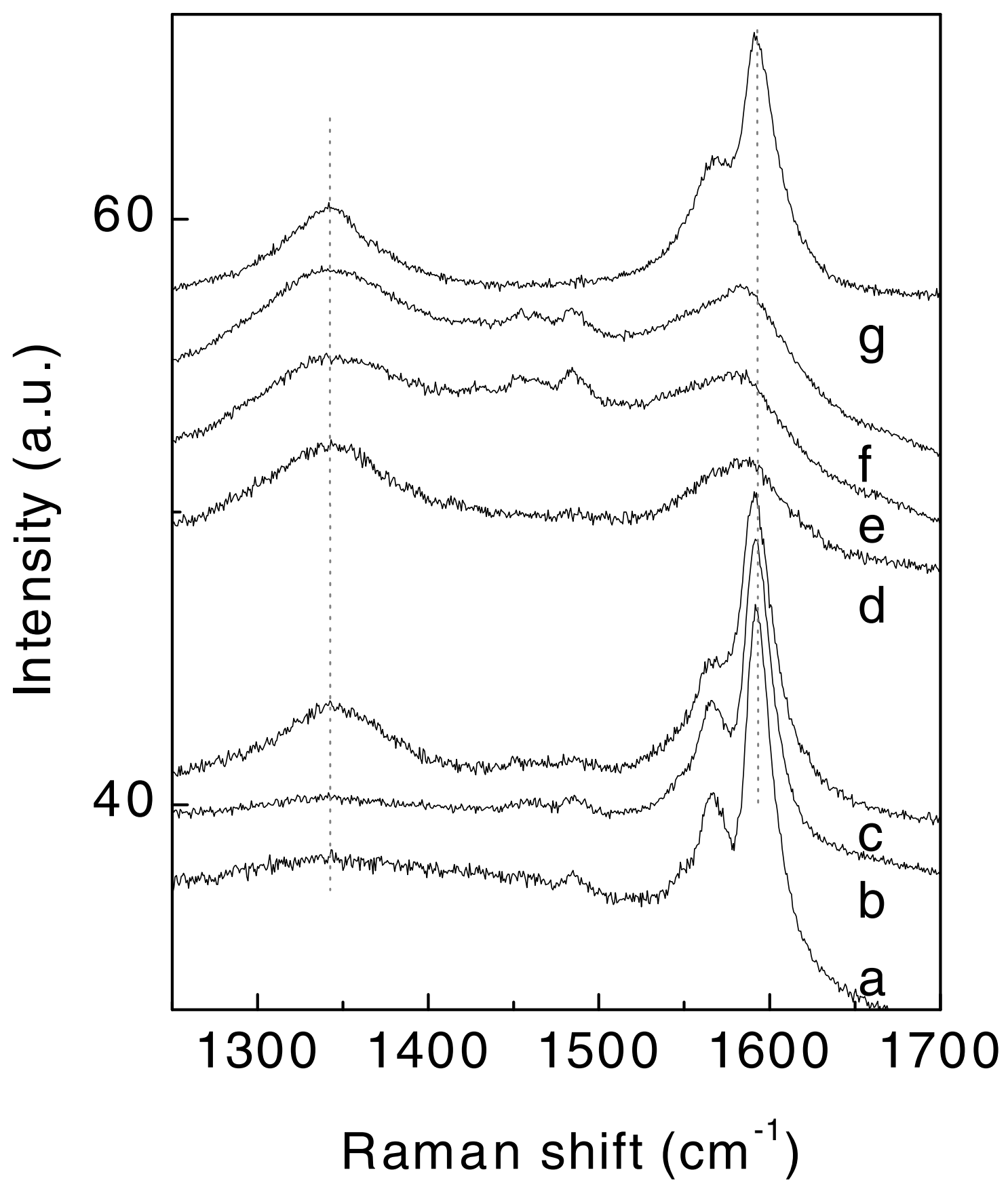

